The Astrophysical JournaL, 530:916-922, 2000 February 20

(C) 2000. The American Astronomical Society. All rights reserved. Printed in U.S.A.

\title{
CORRELATED TIMING AND SPECTRAL VARIATIONS OF THE SOFT X-RAY TRANSIENT AQUILA X-1: EVIDENCE FOR AN ATOLL CLASSIFICATION
}

\author{
P. ReIG, ${ }^{1,2}$ M. MÉNDEZ, ${ }^{3,4}$ M. VAN DER Klis, ${ }^{3}$ AND E. C. ForD ${ }^{3}$ \\ Received 1999 May 19; accepted 1999 October 6
}

\begin{abstract}
Based on Rossi $X$-Ray Timing Explorer data, we discuss the classification of the soft X-ray transient Aquila X-1 in the Z/atoll scheme and the relation of its kilohertz quasi-periodic oscillations (kHz QPOs) properties to the X-ray colors. The color-color diagram shows one elongated ("banana") structure and several "islands" of data points. The power spectra of the island are best represented by a broken power law whereas those of the banana by a power law below $\sim 1 \mathrm{~Hz}$ plus an exponentially cutoff component at intermediate frequencies $(30-60 \mathrm{~Hz})$. The parameters of these two components change in correlation with the position of the source in the color-color diagram. Based on the pattern that the source shows in the color-color diagram and its aperiodic variability, we conclude that Aquila X-1 is an atoll source. We have also investigated the possible correlation between the frequency of the $\mathrm{kHz}$ QPOs and the position of the source in the color-color diagram. The complexity seen in the frequency versus count rate diagram is reduced to a single track when the frequency is plotted against hard or soft color.
\end{abstract}

Subject headings: accretion, accretion disks — stars: individual (Aquila X-1) — stars: neutron X-rays: stars

\section{INTRODUCTION}

The X-ray burst source and soft X-ray transient Aquila $\mathrm{X}-1$ displays $\mathrm{X}$-ray variability on all timescales. Usually the source is in its quiescent state with a very low X-ray luminosity, typically $\sim 10^{33} \mathrm{ergs}^{-1}$ (Verbunt et al. 1994). Within intervals of months to years, it shows outbursts characterized by a gradual increase in flux to a level that sometimes is comparable to the Crab pulsar but at other times is 2 orders of magnitude fainter. The periodicity of these long-term outbursts is unstable with recurrent periods of $\sim 125$ and 309 days (Priedhorsky \& Terrell 1984; Kitamoto et al. 1993). The rise time is typically 10 days; the source remains at a flux maximum for 5-10 days and then decays back to quiescence in typically 30-50 days (Campana et al. 1998; Kitamoto et al. 1993). The spectrum is soft, and no regular pulsations have been detected (Cui et al. 1998; Zhang, Yu, \& Zhang 1998a). During outbursts, Aquila X-1 displays type I X-ray bursts (Zhang et al. 1998a) that can be explained in terms of runaway thermonuclear burning of matter on the surface of the neutron star. As in many other low-mass X-ray binaries (LMXBs), kilohertz quasi-periodic oscillations ( $\mathrm{kHz}$ QPOs) have been detected in the persistent flux of Aquila X-1 (Zhang et al. 1998a; Cui et al. 1998). These $\mathrm{kHz}$ QPOs occurred in the frequency range $740-830 \mathrm{~Hz}$, had a $Q$-value $\left(=v_{\mathrm{QPO}} / \Delta v_{\mathrm{QPO}}\right)$ of over 100 , and had an average fractional rms amplitude of $7 \%$. $\mathrm{kHz}$ QPOs may be caused by orbital motion of gas around the neutron star very close to its surface (see van der Klis 1999 for a recent review).

Recent deep optical and infrared images and lowresolution spectroscopy of this system suggest that the

\footnotetext{
${ }^{1}$ Postal address: Foundation for Research and Technology-Hellas, 711 10 Heraklion, Crete, Greece.

${ }^{2}$ Postal address: Physics Department, University of Crete, 71003 Heraklion, Crete, Greece.

${ }^{3}$ Postal address: Astronomical Institute "Anton Pannekoek," University of Amsterdam and Center for High-Energy Astrophysics, Kruislaan 403, NL-1098 SJ Amsterdam, the Netherlands.

${ }^{4}$ Postal address: Facultad de Ciencias Astronómicas y Geofísicas, Universidad Nacional de La Plata, Paseo del Bosque S/N, 1900 La Plata, Argentina.
}

mass-donating companion is a $V=21.6 \mathrm{~K} 7 \mathrm{~V}$ star, located at an estimated distance of $2.5 \mathrm{kpc}$ (Callanan, Filippenko, \& García 1999; Chevalier et al. 1999) and not the $V=19.2 \mathrm{~K} 1$ IV star 0.48 west, previously assumed to be the candidate (Chevalier \& Ilovaisky 1991; Shahbaz, Casares, \& Charles 1997). At radio wavelengths, Aquila X-1 is unusual in being one of the only neutron star binaries to exhibit radio emission (Hjellming \& Han 1995).

The late-type optical counterpart and the episodic outbursts define Aquila X-1 as a soft X-ray transient (SXT), whereas the existence of type I bursts denotes a low magnetic field neutron star companion (as opposed to black hole). Prior to EXOSAT, LMXBs were classified on the basis of their X-ray luminosity (Schulz, Hasinger, \& Trümper 1989) into two classes: high- and low-luminosity systems. The low-luminosity systems included the so-called $\mathrm{X}$-ray bursters. Thanks to its wide orbit and large collecting area, the EXOSAT satellite allowed long, uninterrupted observations and detailed X-ray timing analysis. Taking into account the rapid aperiodic variability, it turned out that LMXBs could be divided into two different subclasses, $\mathrm{Z}$ and atoll sources, defined by the patterns that these sources display in X-ray color-color diagrams and the properties of the rapid X-ray variability (Hasinger \& van der Klis 1989, hereafter HK89). The classification of Aquila $\mathrm{X}-1$ in this scheme is not certain. Rossi $X$-Ray Timing Explorer ( $R X T E)$ data has suggested that Aquila $\mathrm{X}-1$ is an atoll source (Cui et al. 1998), though some properties appear anomalous. In this paper we investigate the correlated $\mathrm{X}$-ray timing and spectral variations of Aquila X-1 and present evidence for its classification as an atoll source.

\section{OBSERVATIONS}

The data used in this work were retrieved from the public $R X T E$ archive and correspond to two different sets of observations. The first one took place between 1997 February 16 and March 10 and consists of 12 separate observations (one every 2 days, roughly), with a total usable time of about $96 \mathrm{ks}$ (see Zhang et al. 1998a). These data correspond to the decay phase of an outburst. The observations 
during the second set, 1997 August 11-September 10, were conducted at a typical rate of once or twice per day and produced approximately $168 \mathrm{ks}$ of usable data. These observations began halfway through the rising phase of another outburst and finished halfway through the decay phase. Five type I X-ray bursts (two of them were already reported in Zhang et al. 1998a) as well as kHz QPOs were present in the data. The bursts were excluded from our analysis. Another four snapshots taken during the rising phase of the 1998 February-March outburst (Cui et al. 1998) were also analyzed in order to compare our results with those of other authors.

$R X T E$ carries two pointed instruments, the Proportional Counter Array (PCA) developed to cover the lower part of the energy range $(2-60 \mathrm{keV})$ and the High Energy X-ray Timing Experiment (HEXTE) sensitive to X-rays between 15 and $250 \mathrm{keV}$. These instruments are equipped with collimators yielding an FWHM of $1^{\circ}$. In addition, $R X T E$ carries an All-Sky Monitor (ASM) that scans about $80 \%$ of the sky every orbit. In this work we analyzed data from the PCA only since its large collecting area $\left(\sim 6500 \mathrm{~cm}^{2}\right)$ makes it the most appropriate instrument for timing studies.

\section{ANALYSIS AND RESULTS}

\subsection{Color-Color Diagram}

Background-subtracted light curves corresponding to the energy ranges $2.0-3.5 \mathrm{keV}, 3.5-6.0 \mathrm{keV}, 6.0-9.7 \mathrm{keV}$, and 9.7-16.0 keV were used to define the soft and hard colors as $\mathrm{SC}=3.5-6.0 / 2.0-3.5$ and $\mathrm{HC}=9.7-16.0 / 6.0-9.7$, respectively. In a few of the observations, one or two of the five detectors of the PCA were switched off; we only used the three detectors which were always on to calculate these count rates and normalized the count rates to five detectors. The color-color diagram of Aquila X-1 is shown in Figure 1.

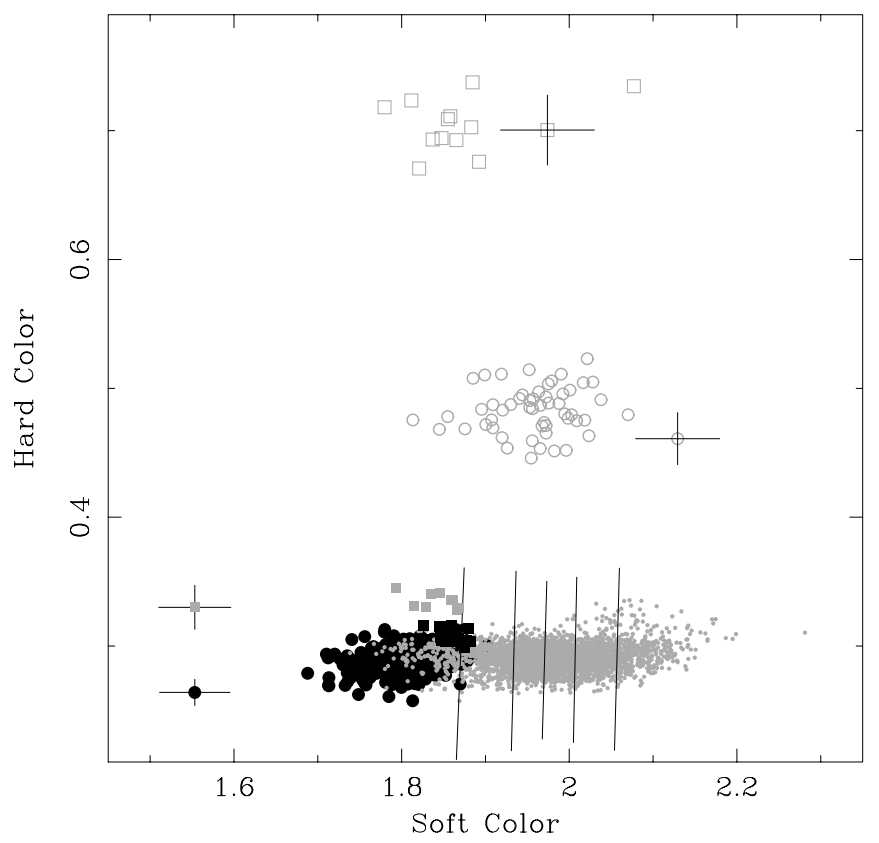

FIG. 1.-Color-color diagram of Aquila X-1. The soft and hard colors are defined as the ratio of count rates in the bands $3.5-6.0 \mathrm{keV}$ and $2.0-3.5$ $\mathrm{keV}$, and $9.7-16.0 \mathrm{keV}$ and $6.0-9.7 \mathrm{keV}$, respectively. The contribution of the background has been subtracted, but no dead-time correction was applied to the data (the dead-time effects on the colors are less than $1 \%$ ). Each point in the banana branch (filled circles) and in the island with the lowest hard color (filled squares) represents $64 \mathrm{~s}$ of data, $128 \mathrm{~s}$ in the middle island (open circles), and $768 \mathrm{~s}$ in the extreme island (open squares). We show the typical error bars in the banana and the island states. Black and gray symbols indicate segments with and without $\mathrm{kHz}$ QPOs, respectively. Vertical lines define the regions into which the banana branch was divided.

TABLE 1

Power Spectral Parameters

\begin{tabular}{|c|c|c|c|c|c|c|c|c|}
\hline \multicolumn{9}{|c|}{ ISLAND State } \\
\hline$S_{a}{ }^{\mathrm{a}}$ & $\mathrm{rms}^{\mathrm{b}}$ & $\alpha$ & $\mathrm{rms}^{\mathrm{b}}$ & $\alpha_{1}{ }^{c}$ & $v_{\mathrm{br}}^{\mathrm{d}}$ & $\alpha_{2}{ }^{\mathrm{e}}$ & Count Rate ${ }^{\mathrm{f}}$ & $\chi_{v}^{2}(\mathrm{dof})$ \\
\hline $1.1 \ldots \ldots \ldots \ldots \ldots \ldots$ & $\ldots$ & $\ldots$ & $34 \pm 2$ & $0.18_{0.25}^{0.16}$ & $0.14 \pm 0.05$ & $0.63 \pm 0.04$ & 45 & $1.23(82)$ \\
\hline $1.5 \ldots \ldots \ldots \ldots \ldots \ldots \ldots$ & $\cdots$ & $\cdots$ & $18.4 \pm 0.5$ & $-0.01 \pm 0.06$ & $15.7_{-1.6}^{+0.6}$ & $1.22 \pm 0.11$ & 200 & $0.79(82)$ \\
\hline 1998 March $2 . . . .$. & $<1.6$ & 1.5 (fixed) & $6.0 \pm 0.3$ & $0.06 \pm 0.10$ & $43_{-4}^{+12}$ & $1.9 \pm 0.6$ & 1481 & $0.80(112)$ \\
\hline \multicolumn{9}{|c|}{ BANANA State } \\
\hline & \multicolumn{2}{|c|}{ VLFN } & \multicolumn{4}{|c|}{ HFN } & & \\
\hline$S_{a}{ }^{a}$ & $\mathrm{rms}^{\mathrm{g}}$ & $\alpha$ & $\mathrm{rms}^{\mathrm{b}}$ & $\alpha$ & $v_{\text {cut }}$ & & Count Rate ${ }^{\mathrm{f}}$ & $\chi_{v}^{2}(\mathrm{dof})$ \\
\hline $2.32 \pm 0.10 \ldots \ldots \ldots$ & $5.6 \pm 0.2$ & $1.61 \pm 0.03$ & $3.5 \pm 0.1$ & $-0.7 \pm 0.2$ & $35_{-7}^{+9}$ & $\ldots$ & 1166 & $1.67(81)$ \\
\hline $2.60 \pm 0.08 \ldots \ldots \ldots$ & $7.2 \pm 0.2$ & $1.59 \pm 0.02$ & $3.0 \pm 0.1$ & $-0.6 \pm 0.2$ & $31_{-6}^{+8}$ & $\begin{array}{l}\cdots \\
\cdots\end{array}$ & 1629 & $1.99(81)$ \\
\hline
\end{tabular}

NoTE.-In the island state, the HFN is represented by a broken power law; in the banana state, the HFN is represented by a power law with an exponential cutoff (see text). Errors are based on a scan in $\chi^{2}$ space using $\Delta \chi^{2}=1$.

${ }^{\text {a }}$ Refers to the regions defined in the color-color diagram (see text).

b $1-100 \mathrm{~Hz}$ rms amplitude (\%).

c Broken power law; slope below the break.

${ }^{d}$ Broken power law; break frequency $(\mathrm{Hz})$.

e Broken power law; slope above the break.

${ }^{\mathrm{f}}$ Background-subtracted count rate for the full PCA (counts s ${ }^{-1}$ ).

g $0.001-1 \mathrm{~Hz}$ rms amplitude (\%). 
The data points in this color-color diagram fall into several distinct groups, the hard color being the defining quantity. The statistical scatter in the uppermost (open squares) and middle (open circles) groups in Figure 1 is considerable. These groups of points come from observations at the very end (after March 5) of the decay of the 1997 March outburst. The lower branch corresponds to observations from the rest of the 1997 February-March run and from the entire 1997 August-September run. The points represented with filled squares correspond to the 1998 March 2 observation. The mean PCA intensity of each group is given in Table 1. At the rising part of the 1997 August-September outburst, the source moved smoothly from left to right along the lower elongated branch and moved back from right to left during the decay phase. During the 1997 February-March outburst, the source also moved toward lower soft colors as the intensity decreased.

In order to measure the source luminosity in the different states spanned in the color-color diagram, we extracted energy spectra and fitted an absorbed blackbody plus power-law model to the data. An iron line at $6.4 \mathrm{keV}$ was added if necessary. The $2-10 \mathrm{keV} \mathrm{X}$-ray luminosity, assuming a distance of $2.5 \mathrm{kpc}$, varies from $2.0 \times 10^{34} \mathrm{ergs}$ $\mathrm{s}^{-1}$ in the uppermost group to $3.2 \times 10^{35} \mathrm{ergs} \mathrm{s}^{-1}$ in the middle one. The elongated lower group of points presents the highest count rate. Here the source luminosity increases from left to right, from $2.4 \times 10^{36}$ to $3.9 \times 10^{36} \mathrm{ergs} \mathrm{s}^{-1}$.

These structures in the color-color diagram and associated count rate differences are similar to those of typical atoll sources. The upper and middle group of points can be associated with the so-called island state, whereas the more elongated lower part would then represent the banana state. By only looking at the position occupied by the points of the 1998 observation, it is not possible to tell whether they define an island or a banana state, and an analysis in terms of the aperiodic variability is needed. In any case they seem to indicate that the transition between the two spectral states is smooth. The gap between the branches is probably observational: no data were obtained for 2 days between the banana and the island and for 3 days between the island and the extreme island states during the 1997 observations.

In order to investigate the variability of Aquila X-1 as a function of the position in the color-color diagram, we divided the color-color plane into several regions as shown in Figure 1. The two island branches define the first two groups. The banana branch was divided so that each region contains approximately the same amount of data. We then approximated the shape of the banana branch with a spline and used the parameter $S_{a}$ to measure positions along this spline (Méndez et al. 1999). We set $S_{a}$ to 2 at (SC, HC) $=$ $(1.732,0.338)$ and to 3 at $(\mathrm{SC}, \mathrm{HC})=(2.045,0.320)$. The intermediate positions are obtained through spline interpolation between the two defining values. This could be done for the banana branch only since the discontinuity between the island and banana branches does not allow one to define a unique path. We arbitrarily assigned the values 1.1 and 1.5 to the two island states. In this way each one of the regions in which we had divided the colorcolor diagram is then characterized by a value of the parameter $S_{a}$.

\subsection{Noise Components}

In order to study the source variability at low frequencies $(\leq 512 \mathrm{~Hz}$ ), we divided the $2-60 \mathrm{keV}$ PCA light curve (no energy selection was done) of each observation into $256 \mathrm{~s}$ segments and calculated the Fourier power spectrum of each segment up to a Nyquist frequency of $1024 \mathrm{~Hz}$. The high-frequency end (950-1024 Hz) of the power spectra was used to determine the underlying Poisson noise, which was subtracted before performing the spectral fitting. The power spectra were normalized to fractional rms squared per Hertz (van der Klis 1995). In a few observations, one or two of the five PCA units were switched off. We have used only those observations for which the five detector units were switched on. This implied a loss of $\sim 11 \%$ of the data. To avoid contributions to the power from the $\mathrm{kHz}$ QPOs, we restricted the spectral fitting to the frequency interval $(1 / 256)-512 \mathrm{~Hz}$. The $256 \mathrm{~s}$ power spectra were then grouped according to the position of the source in the color-color diagram: for each region in the banana and each island state, one mean power spectrum was obtained.

The power spectra were fitted using two broad noise components called the very low frequency noise (VLFN) and the high-frequency noise (HFN). These two components are mathematically represented by a power law and a power law times an exponential cutoff, respectively (HK89). The VLFN accounts for the low-frequency part of the spectrum, whereas the HFN dominates at higher frequencies. Figure 2 shows six power spectra corresponding to different positions in the color-color diagram; all the island states and the lower, middle, and upper banana states are shown.

While the VLFN plus HFN model can satisfactorily describe the banana-state power spectra, it does not provide good fits for the faintest $\left(S_{a}=1.1\right)$ of the two island-state power spectra. Ford \& van der Klis (1998) used a broken power law plus one low-frequency $(10-50 \mathrm{~Hz})$ Lorentzian, possibly representing Lense-Thirring precession (Stella \& Vietri 1998), to fit the island-state power spectra of the atoll source $4 \mathrm{U} 1728-34$. In Aquila X-1 we find that a simple broken power law gives good fits $\left(\chi_{v}^{2} \leq 1.2\right)$ to the power spectra in both island states. Although described by different mathematical functions, the cutoff power law of the banana state and the broken power law of the island state are likely to represent the same type of noise component, namely HFN. To be consistent, we have used the broken power-law model to fit the power spectra of the island states. Figure 3 shows the variation of the amplitude (as fractional rms) of the VLFN (circles), HFN (squares), and broken power-law (triangles) components. The latter refers to the island states.

Unlike the HFN component, the amplitude of the VLFN component does not show any clear trend but remains at $\sim 6 \%-7 \% \mathrm{rms}$, irrespective of the position that the source occupies in the banana. The maximum strength of the HFN is found during the island phases ( $\mathrm{rms}=34 \%$ and $18 \%$ for the upper and lower island states, respectively). This is in contrast to the results of Cui et al. (1998), who did not detect such an HFN component in the first observations of the 1998 February-March outburst (MJD 50,875-50,877) despite the inference from the color diagrams that these data were in an island state. These authors discuss the absence of an HFN in the island state as an unusual phenomenon. We have reanalyzed the first four observations in the Cui et al. (1998) paper and found that there is, in fact, an HFN component with an rms of $6.0 \% \pm 0.3 \%$ (Fig. 2, Table 1 ) in the first two observations. As expected in the island, there is no VLFN (1.6\% upper limit, $95 \%$ confidence level). 


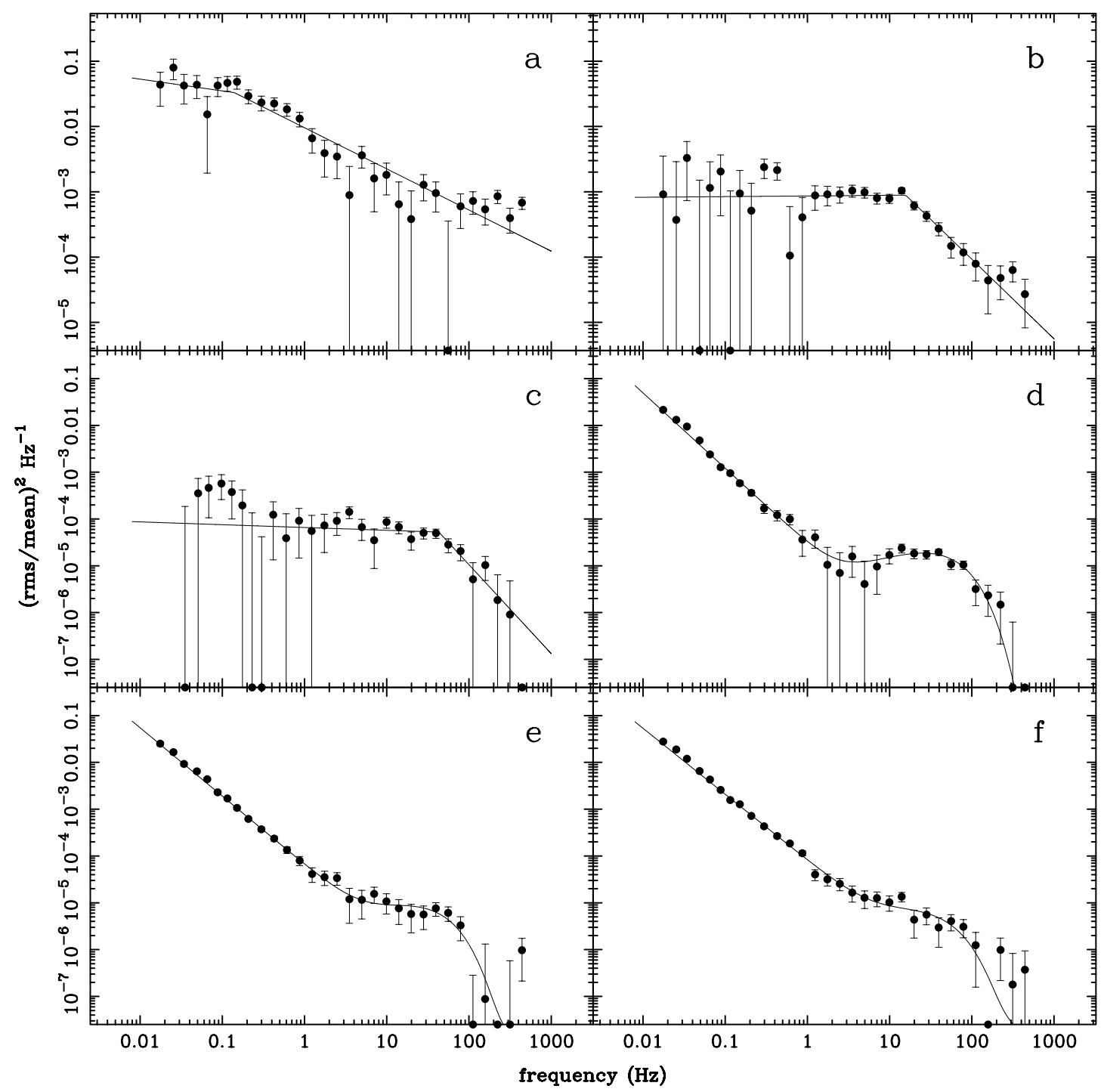

FIG. 2.-Power spectra with best fits for six different positions in the color-color diagram: (a) extreme island, (b) island, (c) lower island (1998 March observation), $(d)$ lower banana $\left(S_{a}=2.32\right)$, $(e)$ middle banana $\left(S_{a}=2.82\right)$, and $(f)$ upper banana $\left(S_{a}=3.04\right)$; see Table 1 . The Poisson level has been subtracted.

The mean hard and soft colors of the corresponding points are $\mathrm{HC}=1.84, \mathrm{SC}=0.34$ and $\mathrm{HC}=1.86, \mathrm{SC}=0.31$ for the first and second observations, respectively. That is, in our color-color diagram, they lie between the lower island and the banana states. This distribution of the island points in the color-color diagram suggests a continuous transition between the island and banana groups. The other two observations produced points in the banana only.

\section{3. $\mathrm{kHz}$ QPOs and the Color-Color Diagram}

For the kHz QPO analysis, we produced power spectra using $64 \mathrm{~s}$ data segments and a Nyquist frequency of 2048 $\mathrm{Hz}$. $\mathrm{kHz}$ QPOs were only observed in a specific range in the color-color diagram, namely the lower banana near $S_{a}=$ 2.32 (Fig. 1, filled circles). The $2-60 \mathrm{keV}$ fractional amplitudes of the $\mathrm{kHz}$ QPOs ranged from $\sim 4.5 \%$ to $\sim 11.7 \%$. We did not detect QPOs in the upper part of the banana with a $95 \%$ confidence upper limit of $1.4 \% \mathrm{rms}$ or in the island with an upper limit of $\sim 8 \%$.

Figure 4 shows the dependence of the frequency of the QPOs as a function of the $2-16 \mathrm{keV}$ PCA count rate. The two parallel groups at the lower left of the plot (open circles, gray circles) correspond to the 1997 February-March observations (Zhang et al. 1998b), while the rest come from the 1997 August-September set of observations.

Méndez et al. (1999) found that there is a much better correlation between the frequency of the $\mathrm{kHz}$ QPOs in $4 \mathrm{U}$ $1608-52$ and the position of the source on the color-color diagram than between frequency and flux. Similarly, the multivalued dependence of $v_{\mathrm{QPO}}$ on X-ray flux in Aquila X-1 is reduced to a single relation when $v_{\mathrm{QPO}}$ is plotted against the $S_{a}$ parameter, which in our case is just a measure of the soft color (Fig. 5). The hard color is not as sensitive to changes in the $\mathrm{kHz}$ QPO frequency in Aquila X-1 as it is in 4U 1608-52 (Kaaret et al. 1998; Méndez et al. 1999).

\section{DISCUSSION}

We have measured the color and timing properties of the LMXB Aquila X-1 and found a behavior similar to that of other low-luminosity LMXBs. The color-color diagram shows the classical atoll shape with the banana and island states. The island state is not defined by a single group of 


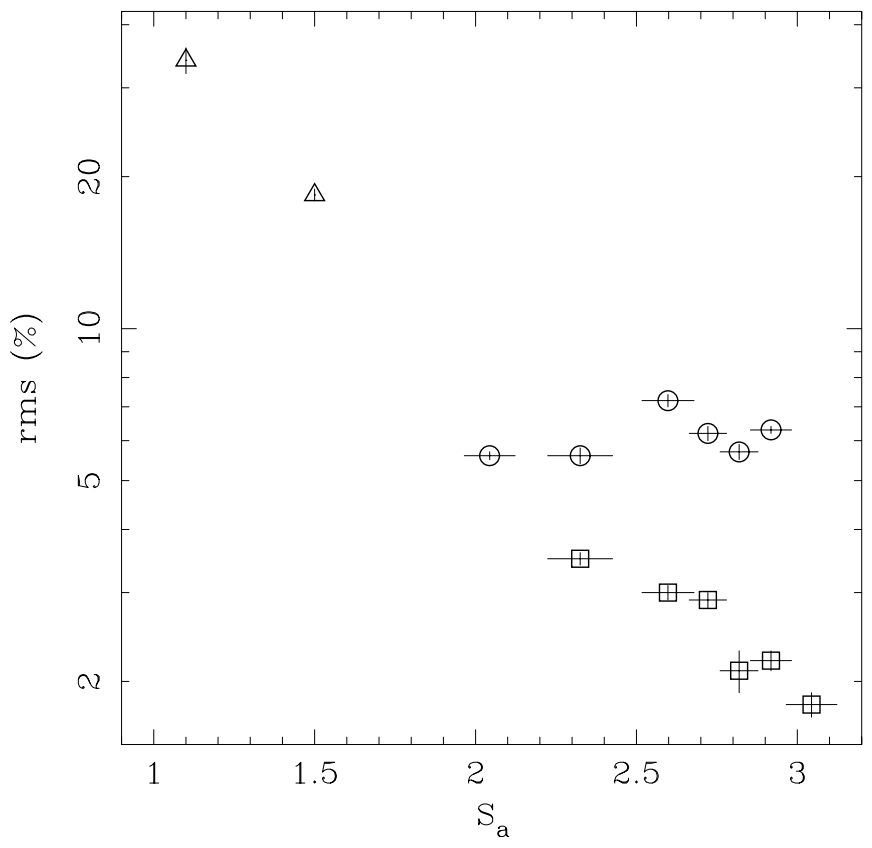

FIG. 3--VLFN (circles) and HFN (squares, triangles) fractional rms $\left(1-100 \mathrm{~Hz}\right.$, full energy band) as a function of the $S_{a}$ parameter (see text). The two triangles correspond to the island and extreme island state and were obtained using a broken power-law function. The $S_{a}$ values for these two points were arbitrarily chosen.

points, but it is split into a number of different groups as in other atoll sources, e.g., 4U 1636-53 (Prins \& van der Klis 1997) and 4U 1608-52 (Yoshida et al. 1993). The lowest count rate and fluxes are detected in the island state with the hardest color and increase as the hard color decreases.

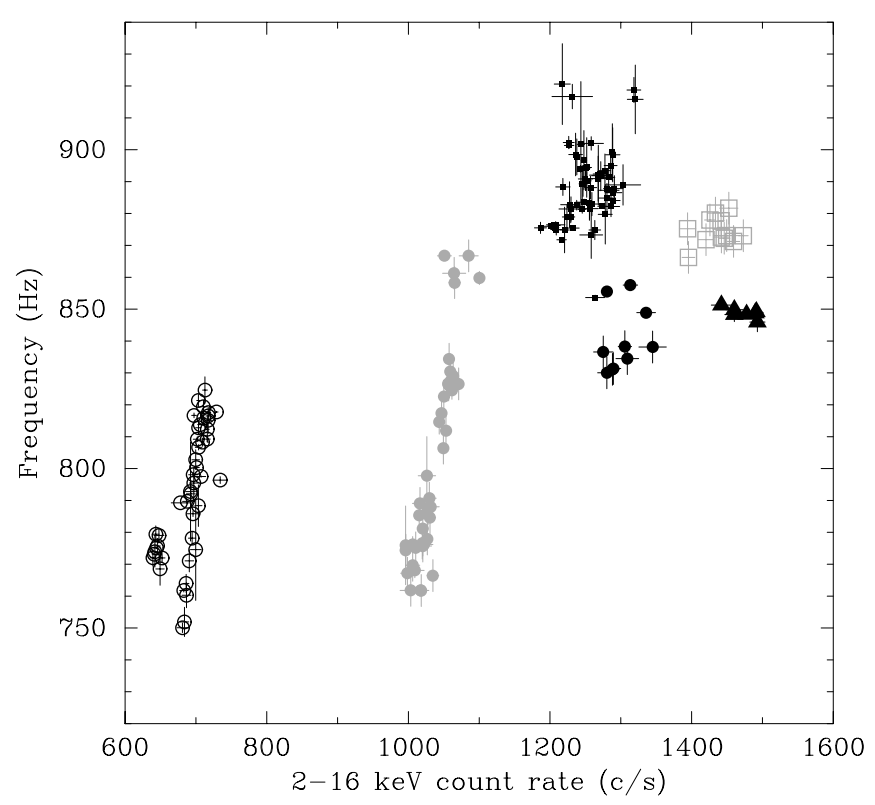

FIG. 4.- Relation between the frequency of the lower $\mathrm{kHz}$ QPO and the $2-16 \mathrm{keV}$ count rate. The count rates have been corrected for background. Each point represents a $128 \mathrm{~s}$ segment. Different symbols represent different observing times as follows: MJD 50,506.245-50,506.358 (gray circles), MJD 50,508.898-50,508.993 (open circles), MJD 50,673.00450,673.473 (filled circles), MJD 50,675.793-50,675.809 (open squares), MJD 50,677.254-50,677.262 (filled triangles), and MJD 50,697.519-50,697.684 ( filled squares).

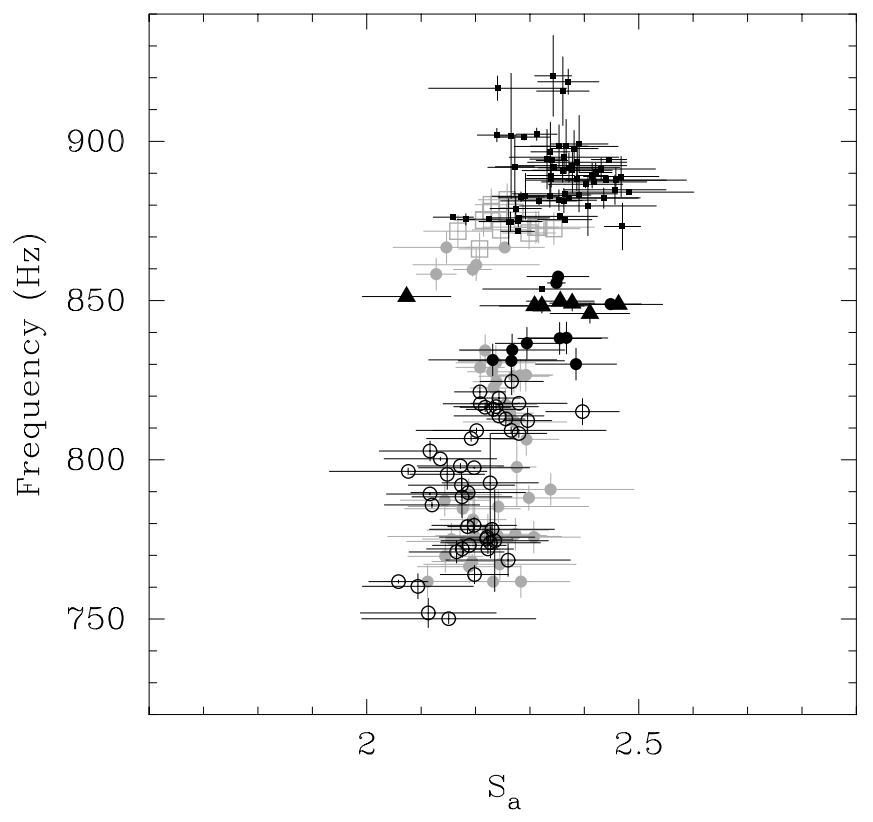

FIG. 5.- Relation between the frequency of the lower $\mathrm{kHz}$ QPOs and $S_{a}$ (see Fig. 1) for the same segments and symbols as those shown in Fig. 4.

Based on the similarity of the color-color diagram of Aquila X-1 to that of other atoll sources, it seems likely that Aquila X-1 can be placed in the group of atoll sources. However, the information provided by the color-color diagram is, by itself, not always sufficient to classify the source state. It is not always possible to make a distinction between $\mathrm{Z}$ and atoll sources or between the two spectral states (island/banana) within the atoll class (HK89). Moreover, in some atoll sources the island and banana branches have been seen to shift in the color-color diagram, in both soft and hard colors (Prins \& van der Klis 1997). The information obtained from the analysis of the noise components in the power spectra provides the key for an unambiguous classification.

The preliminary classification of Aquila X-1 as an atoll source on the basis of the color-color diagram is confirmed by the fast-timing analysis. The power spectra of atoll sources (HK89) are characterized by two broad noise components called the very low frequency noise and the highfrequency noise. The relative strengths of these two components vary in anticorrelation with each other and with the inferred mass accretion rate, $\dot{M}$, as measured by $S_{a}$ in our analysis: the VLFN component appears at the highest inferred $\dot{M}$, whereas that of the HFN decreases as $\dot{M}$ increases. These two components are present in the power spectra of Aquila X-1 (see Fig. 2 and Table 1). As is commonly seen in atoll sources, the VLFN is most prominent in the banana state, while the HFN dominates the island state. The fractional amplitude of the HFN component decreases as the system moves from the island state to the lower banana and from here to the upper banana. The VLFN component is practically undetectable $(\mathrm{rms} \leq 2 \%)$ in the island state, and although present in the banana state, its amplitude does not change as expected (see below). Another similarity between the power spectra of Aquila X-1 in the banana state and those of atoll sources is the presence of wiggles in the VLFN (HK89).

The increase of the break frequency as the X-ray count rate (or flux) increases and the fact that the largest fractional 
amplitude and the hardest spectrum are observed in the two island states represent further evidence in favor of an atoll classification for Aquila X-1 (e.g., 4U 0614 + 09; Méndez et al. 1997). It is worth noting that all these characteristics are also seen in black hole candidates during the low state, emphasizing the observational similarities between atoll sources and black holes systems (van der Klis 1994).

One of the open questions in atoll sources is whether the transition from the island to the banana states occurs continuously or abruptly, that is, with the source jumping from one state to the other. The trends of the spectral parameters just described seem to indicate that such transition is continuous. This idea would be supported by the 1998 March data (Cui et al. 1998). The upper limit to the rms of the VLFN component in the 1998 March observation $(<1.6 \%$, $95 \%$ confidence) is lower than the value in the banana $(5 \%$ $7 \%$ ), and the rms of the HFN is larger (6\% compared to $3.5 \%$ ). The break frequency $v_{\mathrm{br}} \approx 43 \mathrm{~Hz}$ follows the same trend as the other two island states, namely, increases as the source moves into the banana.

An interesting difference between the island state of 1998 March and the other two island states is the source count rate. While the two 1997 March islands show the lowest PCA intensity, the 1998 March one is comparable to that detected in the lower banana state. It is worth noting that the 1997 island data were collected during the decay of an X-ray outburst, whereas that of 1998 correspond to the rise of the outburst. A hysteresis effect may be present.

Our observed VLFN amplitude does not change significantly in the banana state, contrary to what is observed in other atoll sources. Also, the power-law index of the VLFN becomes less steep as the source moves up in the banana branch, while usually in other atoll sources a slight increase, if any change, occurs. This unusual behavior of the VLFN component may be related to nuclear burning on the surface of the neutron star. In periodic X-ray bursters, like Aquila $\mathrm{X}-1$, the entire surface of the star is rapidly $(\leq 10 \mathrm{~s})$ burned by a fast-propagating thermonuclear instability. This can only happen at low mass accretion rates, where the envelope is convectively combustible most of the time. The convective burning makes it difficult for slower combustion to occur, thus suppressing the VLFN (Bildsten 1993). Yu et al. (1999) reported another unusual aspect of the VLFN in Aquila X-1, namely, its disappearance after a type I burst, in which the $2-10 \mathrm{keV}$ flux decreased by about $10 \%$ and the $\mathrm{kHz}$ QPO frequency fell abruptly by $\sim 37 \mathrm{~Hz}$.

Aquila X-1 is unusual among the group of low-mass X-ray binaries with $\mathrm{kHz}$ QPOs in that only one $\mathrm{kHz}$ QPO has been so far observed. All other sources ( $\mathrm{Z}$ and atoll) have at least sometimes shown two simultaneous $\mathrm{kHz}$ QPOs. Nevertheless, the relationship between the frequency of the $\mathrm{kHz}$ QPOs and the X-ray flux in Aquila X-1 is very similar to that seen in the atoll sources $4 \mathrm{U} 1608-52$ (Méndez et al. 1999) and 4U 0614+091 (Ford et al. 1997): on timescales longer than $\sim 1$ day, there is no correlation between the $\mathrm{kHz}$ frequency and the X-ray intensity (or flux), whereas on short timescales ( $\sim$ hours), these two quantities correlate remarkably well (Zhang et al. 1998a). We also find that similarly to other sources, on timescales longer than a day, the QPO frequency correlates much better to color (or $S_{a}$ ) than to count rate.

\section{CONCLUSION}

We have used RXTE data to carry out a timing analysis with the aim of solving the issue of the classification of Aquila $\mathrm{X}-1$ in the $\mathrm{Z}$ /atoll scheme. Aquila $\mathrm{X}-1$ traces a pattern in the color-color diagram that is consistent with having an island and a banana branch. The PCA intensity correlates with the position of the source in the color-color diagram, decreasing in the sense of upper banana to lower banana to island state.

The power density spectra of the banana states can be described in terms of a power-law component, the so-called very low frequency noise and a cutoff power-law component or high-frequency noise. In the island state, the highfrequency noise component is best described in terms of a broken power law, whereas the very low frequency noise is practically undetectable. The characteristics of the power spectra also change in correlation with the position of the source in the color-color diagram. The amplitude of the high-frequency noise increases as the $S_{a}$ parameter decreases. Finally, we have shown that the soft color (via the $S_{a}$ parameter) correlates with the frequency of the $\mathrm{kHz}$ QPO.

Based on the correlation of the X-ray spectral properties of Aquila X-1 (its position in the color-color diagram) and its fast-timing behavior, we conclude that Aquila X-1 is an atoll source.

This work was supported by the Netherlands Foundation for research in astronomy (ASTRON) under grant 781-76-017, the Netherlands Research School for Astronomy (NOVA), and the NWO Spinoza grant 08-0 to E. P. J. van den Heuvel. P. R. acknowledges support from the European Union through the Training and Mobility Research Network grant ERBFMRX/CT98/0195. M. M. is a fellow of the Consejo Nacional de Investigaciones Científicas y Técnicas de la República Argentina. This research has made use of data obtained through the High Energy Astrophysics Science Archive Research Center Online Service, provided by the NASA/Goddard Space Flight Center.

\section{REFERENCES}

Bildsten, L. 1993, ApJ, 418, L21

Callanan, P. J., Filippenko, A. V., \& García, M. R. 1999, IAU Circ. 7086

Campana, S., Stella, L., Mereghetti, S., Colpi, M., Tavani, M., Ricci, D., Dal Fiume, D., \& Belloni, T. 1998, ApJ, 499, L65

Chevalier, C., \& Ilovaisky, S. A. 1991, A\&A, 251, L11

Chevalier, C., Ilovaisky, S. A., Leisy, P., \& Patat, F. 1999, A\&A, 347, L51

Cui, W., Barret, D., Zhang, S. N., Chen, W., Boirin, L., \& Swank, J. 1998, ApJ, 502, L49

Ford, E. C., Kaaret, P., Chen, K., Tavani, M., Barret, D., Bloser, P., Grindlay, J., Harmon, B. A., Paciesas, W. S., \& Zhang, S. N. 1997, ApJ, 486, L47

Ford, E. C., \& van der Klis, M. 1998, ApJ, 506, L39

Hasinger, G., \& van der Klis, M. 1989, A\&A, 225, 79 (HK89)
Hjellming, R. M., \& Han, X. 1995, in X-Ray Binaries, ed. W. H. G. Lewin, J. van Paradijs, \& E. P. J. van den Heuvel (Cambridge: Cambridge Univ. Press), 308

Kaaret, P., Yu, W., Ford, E. C., \& Zhang, N. S. 1998, ApJ, 497, L93

Kitamoto, S., Tsunemi, H., Miyamoto S., \& Roussel-Dupre, D. 1993, ApJ, 403,315

Méndez, M., van der Klis, M., Ford, E. C., Wijnands, R., \& van Paradijs, J. 1999, ApJ, 511, L49

Méndez, M., van der Klis, M., van Paradijs, J., Lewin, W. H. G., Lamb, F. K., Vaughan, B. A., Kuulkers, E., \& Psaltis, D. 1997, ApJ, 485, L37

Priedhorsky, W. C., \& Terrell, J. 1984, ApJ, 280, 661

Prins, S., \& van der Klis, M. 1997, A\&A, 319, 498

Schulz, N. S., Hasinger, G., \& Trümper, J. 1989, A\&A, 225, 48 


\section{REIG ET AL.}

Shahbaz, T., Casares, J., \& Charles, P. A. 1997, A\&A, 326, L5

Stella, L., \& Vietri, M. 1998, ApJ, 492, L59

van der Klis, M. 1994, ApJS, 92, 511

. 1995, in X-Ray Binaries, ed. W. H. G. Lewin, J. van Paradijs, \&

E. P. J. van den Heuvel (Cambridge: Cambridge Univ. Press), 252

1999, in Third William Fairbank Meeting, in press (preprint astro$\mathrm{ph} / 9812395)$
Verbunt, F., Belloni, T., Johnston, H. M., van der Klis, M., \& Lewin, W. H. G. 1994, A\&A, 285, 903

Yoshida, K., Mitsuda, K., Ebisawa, K., \& Done, C. 1993, PASJ, 45, 605

Yu, W., Li, T. P., Zhang, W., \& Zhang, S. N. 1999, ApJ, 512, L35

Zhang, S. N., Yu, W., \& Zhang, W. 1998a, ApJ, 494, L71

Zhang, W., Jahoda, K., Kelley, R. L., Strohmayer, T. E., Swank, J. H., \& Zhang, S. N. 1998b, ApJ, 495, L9 